TRANSACTIONS OF THE

AMERICAN MATHEMATICAL SOCIETY

Volume 358, Number 5, Pages 2091-2104

S 0002-9947(05)03748-7

Article electronically published on April 22, 2005

\title{
STABLE MAPPING CLASS GROUPS OF 4-MANIFOLDS WITH BOUNDARY
}

\author{
OSAMU SAEKI
}

\begin{abstract}
We give a complete algebraic description of the mapping class groups of compact simply connected 4-manifolds with boundary up to connected sum with copies of $S^{2} \times S^{2}$.
\end{abstract}

\section{INTRODUCTION}

It has been shown in [15] that two orientation preserving homeomorphisms of a closed simply connected 4-manifold are homotopic if and only if the induced automorphisms on the second homology group coincide with each other (see also [4. §5]). Using this, Quinn [15] has shown that two homeomorphisms inducing the same automorphism on the second homology group are actually topologically isotopic (see also [13]). Quinn has also shown that two diffeomorphisms inducing the same automorphism are smoothly isotopic after the connected sum with the identity diffeomorphism of some copies of $S^{2} \times S^{2}$. In this paper we give a similar smooth stable isotopy criterion for the case where the simply connected 4-manifold has nonempty connected boundary.

Let $M$ be a compact 1-connected 4-manifold with nonempty connected boundary. In this paper we first give an algebraic criterion for two diffeomorphisms of $M$ to be stably isotopic (see \$2), where two diffeomorphisms $h_{0}$ and $h_{1}$ of $M$ which are the identity on the boundary are stably isotopic relative to boundary if $h_{0} \sharp k$ (id) and $h_{1} \sharp k(\mathrm{id})$ are smoothly isotopic relative to boundary as diffeomorphisms of $M \sharp k\left(S^{2} \times S^{2}\right)$ for some $k \geq 0$. We will see that certain homomorphisms

$$
H_{2}(M, \partial M ; \mathbf{Z}) \rightarrow H_{2}(M ; \mathbf{Z}),
$$

called variation maps, associated with such diffeomorphisms play an essential role.

Then in $\$ 3$ we introduce the notion of the stable mapping class group of $M$ and give its complete algebraic description using the result of $₫ 2$. The algabraic objects that play an important role here are homomorphisms, called variational homomorphisms, $H_{2}(M, \partial M ; \mathbf{Z}) \rightarrow H_{2}(M ; \mathbf{Z})$ which are defined to be abstract homomorphisms satisfying certain algebraic conditions. We will see that the set of variational homomorphisms form a group with respect to a certain multiplication.

Received by the editors April 4, 2001 and, in revised form, April 25, 2004.

2000 Mathematics Subject Classification. Primary 57N13; Secondary 57R52, 57N37.

Key words and phrases. Isotopy, 4-manifold with boundary, stable mapping class group, variation map, isometry.

The author was supported in part by Grant-in-Aid for Scientific Research (No. 11440022), Ministry of Education, Science and Culture, Japan. 
The main content of $\$ 3$ is a construction of a diffeomorphism which realizes a given variational homomorphism.

In 4 , we study the relationship between the group of variational homomorphisms and the group of isometries of $H_{2}(M)$ endowed with the intersection form, which will be used in $\$ 5$,

In $\$ 5$, we consider the stable mapping class group of boundary-free diffeomorphims of 4-manifolds whose boundaries are certain spherical 3-manifolds.

Throughout the paper, we work in the smooth category unless otherwise specified. All the homology and cohomology groups are with integer coefficients unless otherwise indicated. We use the symbol "" to denote a diffeomorphism between smooth manifolds or an appropriate isomorphism between algebraic objects.

The author would like to thank the referee for drawing his attention to Kreck's paper [10]. He also would like to thank the people at IRMA, Strasbourg, for their hospitality during the preparation of the manuscript.

\section{ISOTOPY OF 1-CONNECTED 4-MANIFOLDS WITH BOUNDARY}

Let $M$ be a compact 1-connected 4-manifold with nonempty connected boundary. We first define the variation map associated with a diffeomorphism of $M$, which will play an important role throughout the paper.

Definition 2.1. Let $h: M \rightarrow M$ be a diffeomorphism which is the identity on the boundary. We define the variation map $\Delta_{h}: H_{2}(M, \partial M) \rightarrow H_{2}(M)$ as follows. For a homology class $\gamma \in H_{2}(M, \partial M)$, take a 2 -cycle $(D, \partial D)$ in $(M, \partial M)$ representing $\gamma$. Then $D \cup(-h(D))$ is a 2-cycle in $M$ and we define $\Delta_{h}(\gamma)$ to be the class represented by $D \cup(-h(D))$. Note that this does not depend on the choice of $(D, \partial D)$ and that $\Delta_{h}$ is a homomorphism. See also 8 .

Note that if two diffeomorphisms of $M$ which are the identity on the boundary are isotopic relative to boundary, then their variation maps coincide with each

other. The main result of this section is that the converse is also true "stably" as follows.

Theorem 2.2. Let $M$ be a compact 1-connected 4-manifold with nonempty connected boundary. Suppose that $h_{0}$ and $h_{1}: M \rightarrow M$ are two diffeomorphisms with $\left.h_{0}\right|_{\partial M}=\left.h_{1}\right|_{\partial M}$ being the identity map. Then $h_{0}$ and $h_{1}$ are stably isotopic relative to boundary if and only if $\Delta_{h_{0}}=\Delta_{h_{1}}: H_{2}(M, \partial M) \rightarrow H_{2}(M)$.

Proof. In the following, for an integer $k \geq 0, M_{k}$ will denote the 4-manifold $M \sharp k\left(S^{2} \times S^{2}\right)$.

If $h_{0}$ and $h_{1}$ are stably isotopic relative to boundary, then it is easy to see that their variation maps coincide with each other, since on the direct summand of $H_{2}\left(M_{k}, \partial M_{k}\right)$ corresponding to $H_{2}\left(\sharp^{k} S^{2} \times S^{2}\right)$, the variation maps of $h_{j} \sharp k(\mathrm{id})$, $j=0,1$, are the zero homomorphisms.

In order to prove the converse, let us consider the following construction, which is called an open book construction (see [8]). For a diffeomorphism $h: M \rightarrow M$ which is the identity on the boundary, let $L_{h}$ be the 5-dimensional manifold with boundary obtained from $M \times[0,1]$ by identifying $M \times\{1\}$ with $M \times\{0\}$ using $h: M \times\{1\} \rightarrow M \times\{0\}$. Note that $\partial L_{h}$ is canonically diffeomorphic to $\partial M \times S^{1}$. Then, let $N_{h}$ be the closed 5-dimensional manifold obtained by attaching $\partial M \times D^{2}$ to $L_{h}$ along the boundary. Note that $N_{h}$ is 1 -connected and that $M$ is naturally identified with $M \times\{0\} \subset N_{h}$. 
Then, by an argument similar to that in $[9, \S 2]$, we easily obtain the following.

Lemma 2.3. A diffeomorphism $h: M \rightarrow M$ which is the identity on the boundary is pseudo-isotopic to the identity relative to boundary if and only if $N_{h}$ bounds a compact 1-connected 6 -dimensional manifold $W$ such that $\pi_{j}(W, M)=0$ for all $j$.

We also have the following.

Lemma 2.4. Let $h: M \rightarrow M$ be a diffeomorphism which is the identity on the boundary. Then we have the following exact sequence:

$$
0 \rightarrow H_{3}\left(N_{h}\right) \rightarrow H_{2}(M, \partial M) \stackrel{\Delta_{h}}{\longrightarrow} H_{2}(M) \rightarrow H_{2}\left(N_{h}\right) \rightarrow 0 .
$$

Proof. Consider the exact sequence for the pair $\left(N_{h}, M\right)$ :

$$
0 \rightarrow H_{3}\left(N_{h}\right) \rightarrow H_{3}\left(N_{h}, M\right) \stackrel{\partial}{\longrightarrow} H_{2}(M) \rightarrow H_{2}\left(N_{h}\right) \rightarrow H_{2}\left(N_{h}, M\right) .
$$

Let $\widetilde{M} \cong M \times[0,1]$ be a normal 1-disk bundle neighborhood of $M$ in $N_{h}$. Note that the closure of $N_{h} \backslash \widetilde{M}$ is again diffeomorphic to $M \times[0,1]$. Hence, by excision together with Poincaré-Lefschetz duality, we have

$$
\begin{aligned}
H_{i}\left(N_{h}, M\right) & \cong H_{i}\left(N_{h}, \widetilde{M}\right) \cong H_{i}(M \times[0,1], \partial(M \times[0,1])) \\
& \cong H^{5-i}(M \times[0,1]) \cong H^{5-i}(M) \cong H_{i-1}(M, \partial M) .
\end{aligned}
$$

In particular, we have $H_{2}\left(N_{h}, M\right) \cong H_{1}(M, \partial M)=0$, since $M$ is simply connected and $\partial M$ is connected. Furthermore, under the isomorphism $H_{3}\left(N_{h}, M\right) \cong$ $H_{2}(M, \partial M)$ given above, the boundary homomorphism $\partial: H_{3}\left(N_{h}, M\right) \rightarrow H_{2}(M)$ appearing in (2.1) is identified with the variation map $\Delta_{h}: H_{2}(M, \partial M) \rightarrow H_{2}(M)$. This completes the proof of Lemma 2.4

Let us go back to the proof of Theorem 2.2. Let $h_{0}$ and $h_{1}: M \rightarrow M$ be diffeomorphisms which are the identity on the boundary such that $\Delta_{h_{0}}=\Delta_{h_{1}}$. Set $h=h_{0} \circ\left(h_{1}\right)^{-1}$. Then it is easy to show that $\Delta_{h}$ is the zero homomorphism (for example, see [16, §9]). Then by following the same argument as in [9, §2] and by replacing the Wang exact sequence for a bundle over $S^{1}$ with that of Lemma 2.4. we can construct a 6 -dimensional manifold $W$ as in Lemma 2.3. Therefore, $h$ is pseudo-isotopic to the identity relative to boundary (see also the last paragraph of [10]).

Thus $h_{0}$ and $h_{1}$ are pseudo-isotopic relative to boundary. Then by Quinn [15, they are stably isotopic relative to boundary. This completes the proof of Theorem 2.2 .

Compare Theorem 2.2 with [16, Conjecture 9.6].

Remark 2.5. In the above situation, we can prove that $h_{0} \sharp k$ (id) and $h_{1} \sharp k$ (id) are pseudo-isotopic relative to boundary for some $k \geq 0$ also by using an argument based on the theory of open books on 5-dimensional manifolds [16, 17].

\section{STABLE MAPPING CLASS GROUP AND VARIATIONAL HOMOMORPHISMS}

In this section, we define the stable mapping class group of a compact 4-manifold with boundary and give a result which describes it algebraically. 
Definition 3.1. Let $M$ be a compact 4-manifold with boundary. A stable diffeomorphism of $M$ is a diffeomorphism of $M_{k}=M \sharp k\left(S^{2} \times S^{2}\right)$ for some $k$. Two stable diffeomorphisms $h_{0}: M_{k_{0}} \rightarrow M_{k_{0}}$ and $h_{1}: M_{k_{1}} \rightarrow M_{k_{1}}$ which are the identity on the boundary are said to be stably isotopic relative to boundary if $h_{0} \sharp k_{0}^{\prime}(\mathrm{id}): M_{k_{0}+k_{0}^{\prime}} \rightarrow M_{k_{0}+k_{0}^{\prime}}$ and $h_{1} \sharp k_{1}^{\prime}(\mathrm{id}): M_{k_{1}+k_{1}^{\prime}} \rightarrow M_{k_{1}+k_{1}^{\prime}}$ are isotopic relative to boundary for some $k_{0}^{\prime}$ and $k_{1}^{\prime}$ with $k_{0}+k_{0}^{\prime}=k_{1}+k_{1}^{\prime}$. Finally, the set of all stable isotopy classes relative to boundary of stable diffeomorphisms of $M$ which are the identity on the boundary is called the stable mapping class group of $(M, \partial M)$ and is denoted by $\mathcal{S} \mathcal{M}(M, \partial M)$. Note that if we denote by $\mathcal{M}(M, \partial M)$ the usual mapping class group relative to boundary, then we can naturally identify $\mathcal{S M}(M, \partial M)$ with the inductive limit

$$
\lim _{k \rightarrow \infty} \mathcal{M}\left(M_{k}, \partial M_{k}\right)
$$

This obviously forms a group with respect to the composition of maps.

Let $M$ be as above and let $h: M_{k} \rightarrow M_{k}$ be a stable diffeomorphism of $M$ which is the identity on the boundary. Let us denote the variation map of $h$ by $\Delta_{h}: H_{2}\left(M_{k}, \partial M_{k}\right) \rightarrow H_{2}\left(M_{k}\right)$. We also denote the induced map $h_{*}: H_{2}\left(M_{k}\right) \rightarrow$ $H_{2}\left(M_{k}\right)$ by $\Lambda_{h}$. Since the intersection form

$$
H_{2}\left(M_{k}\right) \times H_{2}\left(M_{k}, \partial M_{k}\right) \rightarrow \mathbf{Z}
$$

of $M_{k}$ is unimodular, we may regard $H_{2}\left(M_{k}\right)$ and $H_{2}\left(M_{k}, \partial M_{k}\right)$ dual to each other. For a homomorphism $\rho$ involving these spaces, $\rho^{*}$ will denote its dual homomorphism. Let $j: M_{k} \rightarrow\left(M_{k}, \partial M_{k}\right)$ denote the inclusion map.

In order to give an algebraic description of the stable mapping class group, let us prepare some lemmas.

Lemma 3.2. We have the following:

(1) $\left(j_{*}\right)^{*}=j_{*}: H_{2}\left(M_{k}\right) \rightarrow H_{2}\left(M_{k}, \partial M_{k}\right)$.

(2) $\Lambda_{h}=\mathrm{id}-\Delta_{h} \circ j_{*}: H_{2}\left(M_{k}\right) \rightarrow H_{2}\left(M_{k}\right)$.

(3) $j_{*}=\Lambda_{h}^{*} \circ j_{*} \circ \Lambda_{h}: H_{2}\left(M_{k}\right) \rightarrow H_{2}\left(M_{k}\right)$.

(4) $\Delta_{h}+\Delta_{h}^{*}=\Delta_{h} \circ j_{*} \circ \Delta_{h}^{*}: H_{2}\left(M_{k}, \partial M_{k}\right) \rightarrow H_{2}\left(M_{k}\right)$.

(5) For two stable diffeomorphisms $h$ and $h^{\prime}: M_{k} \rightarrow M_{k}$ which are the identity on the boundary, we have

$$
\Delta_{h \circ h^{\prime}}=\Delta_{h}+\Lambda_{h} \circ \Delta_{h^{\prime}}: H_{2}\left(M_{k}, \partial M_{k}\right) \rightarrow H_{2}\left(M_{k}\right) .
$$

Proof. (1) This follows from the fact that $\alpha \cdot j_{*} \beta=\beta \cdot j_{*} \alpha$ for all $\alpha, \beta \in H_{2}\left(M_{k}\right)$, where "." denotes the intersection form (3.1).

(2) For each $\alpha \in H_{2}\left(M_{k}\right)$, we have

$$
\left(\mathrm{id}-\Delta_{h} \circ j_{*}\right)(\alpha)=\alpha-\left(\alpha-h_{*} \alpha\right)=h_{*} \alpha=\Lambda_{h} \alpha .
$$

Thus the result follows.

(3) This follows from the fact that $h_{*}: H_{2}\left(M_{k}\right) \rightarrow H_{2}\left(M_{k}\right)$ is an isometry of $\left(H_{2}\left(M_{k}\right), \cdot\right)$, where "." denotes the intersection form of $M_{k}$ on $H_{2}\left(M_{k}\right)$.

(4) We have

$$
\begin{aligned}
\Delta_{h}+\Delta_{h}^{*}=\Delta_{h} \circ j_{*} \circ \Delta_{h}^{*} & \Longleftrightarrow\left(\mathrm{id}-\Delta_{h} \circ j_{*}\right) \circ \Delta_{h}^{*}=-\Delta_{h} \\
& \Longleftrightarrow \Lambda_{h} \circ \Delta_{h}^{*}=-\Delta_{h} \\
& \Longleftrightarrow \Delta_{h}^{*}=-\Lambda_{h}^{-1} \circ \Delta_{h},
\end{aligned}
$$


where (3.2) follows from (2). This is equivalent to

$$
\Delta_{h} \beta \cdot \alpha=-\left(\Lambda_{h}^{-1} \circ \Delta_{h} \alpha\right) \cdot \beta
$$

for all $\alpha, \beta \in H_{2}\left(M_{k}, \partial M_{k}\right)$. Let $a$ and $b$ be 2-cycles in $\left(M_{k}, \partial M_{k}\right)$ representing $\alpha$ and $\beta$, respectively. Then the right-hand side of the above equation is equal to

$$
\begin{aligned}
-h^{-1}(a-h a) \cdot b & =\left(a-h^{-1} a\right) \cdot b \\
& =a \cdot b-h^{-1} a \cdot b \\
& =b \cdot a-h b \cdot a \\
& =(b-h b) \cdot a .
\end{aligned}
$$

Thus we have (3.4) and have proved the required equality.

(5) For every 2-cycle $a$ of $\left(M_{k}, \partial M_{k}\right)$, we have

$$
a-\left(h \circ h^{\prime}\right) a=a-h a+h a-h\left(h^{\prime} a\right)=a-h a+h\left(a-h^{\prime} a\right) .
$$

Thus we have

$$
\Delta_{h \circ h^{\prime}}=\Delta_{h}+\Lambda_{h} \circ \Delta_{h^{\prime}}
$$

This completes the proof.

Definition 3.3. Let $M$ be a compact 4-manifold with boundary. A homomorphism $\Delta: H_{2}(M, \partial M) \rightarrow H_{2}(M)$ is variational if $\Delta+\Delta^{*}=\Delta \circ j_{*} \circ \Delta^{*}$. Let $\mathcal{V}(M, \partial M)$ denote the set of all variational homomorphisms.

Lemma 3.4. Let $\Delta: H_{2}(M, \partial M) \rightarrow H_{2}(M)$ be a variational homomorphism. Then $\Lambda=\mathrm{id}-\Delta \circ j_{*}$ is an isometry of the inner product space $\left(H_{2}(M), \cdot\right)$, where "." denotes the intersection form of $M$ on $\mathrm{H}_{2}(M)$.

Proof. Since $\Delta+\Delta^{*}=\Delta \circ j_{*} \circ \Delta^{*}$ holds by our assumption, we have $j_{*} \circ\left(\Delta+\Delta^{*}\right)=$ $j_{*} \circ \Delta \circ j_{*} \circ \Delta^{*}$. This is equivalent to the equality

$$
\left(\mathrm{id}-j_{*} \circ \Delta\right) \circ\left(\mathrm{id}-j_{*} \circ \Delta^{*}\right)=\mathrm{id}: H_{2}(M) \rightarrow H_{2}(M) \text {. }
$$

Since $H_{2}(M)$ is free, we see that id $-j_{*} \circ \Delta$ and $\Lambda^{*}=\mathrm{id}-j_{*} \circ \Delta^{*}$ are isomorphisms which are inverses to each other. Thus $\Lambda$ is an isomorphism and

$$
\left(\mathrm{id}-j_{*} \circ \Delta^{*}\right) \circ\left(\mathrm{id}-j_{*} \circ \Delta\right)=\mathrm{id}
$$

also holds. In particular, we have

$$
\left(\mathrm{id}-j_{*} \circ \Delta^{*}\right) \circ\left(\mathrm{id}-j_{*} \circ \Delta\right) \circ j_{*}=j_{*},
$$

which is equivalent to $\Lambda^{*} \circ j_{*} \circ \Lambda=j_{*}$. Thus $\Lambda$ is an isometry. This completes the proof.

Lemma 3.5. The set $\mathcal{V}(M, \partial M)$ of all variational homomorphisms forms a group under the multiplication given by

$$
\Delta_{1} * \Delta_{2}=\Delta_{1}+\left(\mathrm{id}-\Delta_{1} \circ j_{*}\right) \circ \Delta_{2}
$$

for $\Delta_{1}, \Delta_{2} \in \mathcal{V}(M, \partial M)$.

Proof. Put $\mathcal{V}=\mathcal{V}(M, \partial M)$ for simplicity. Let us first show that $\Delta_{1} * \Delta_{2} \in \mathcal{V}$. Putting $\Lambda_{i}=\mathrm{id}-\Delta_{i} \circ j_{*}, i=1,2$, we have

$$
\begin{aligned}
\left(\Delta_{1} * \Delta_{2}\right)+\left(\Delta_{1} * \Delta_{2}\right)^{*} & =\left(\Delta_{1}+\Lambda_{1} \circ \Delta_{2}\right)+\left(\Delta_{1}+\Lambda_{1} \circ \Delta_{2}\right)^{*} \\
& =\Delta_{1}+\Delta_{1}^{*}+\Lambda_{1} \circ \Delta_{2}+\left(\Lambda_{1} \circ \Delta_{2}\right)^{*} .
\end{aligned}
$$


On the other hand, we have

$$
\begin{aligned}
& \left(\Delta_{1} * \Delta_{2}\right) \circ j_{*} \circ\left(\Delta_{1} * \Delta_{2}\right)^{*} \\
& =\left(\Delta_{1}+\Lambda_{1} \circ \Delta_{2}\right) \circ j_{*} \circ\left(\Delta_{1}+\Lambda_{1} \circ \Delta_{2}\right)^{*} \\
& =\Delta_{1} \circ j_{*} \circ \Delta_{1}^{*}+\Delta_{1} \circ j_{*} \circ \Delta_{2}^{*} \circ \Lambda_{1}^{*}+\Lambda_{1} \circ \Delta_{2} \circ j_{*} \circ \Delta_{1}^{*}+\Lambda_{1} \circ \Delta_{2} \circ j_{*} \circ \Delta_{2}^{*} \circ \Lambda_{1}^{*} \\
& =\left(\Delta_{1}+\Delta_{1}^{*}\right)+\Delta_{1} \circ j_{*} \circ \Delta_{2}^{*} \circ \Lambda_{1}^{*}+\Lambda_{1} \circ \Delta_{2} \circ j_{*} \circ \Delta_{1}^{*}+\Lambda_{1} \circ\left(\Delta_{2}+\Delta_{2}^{*}\right) \circ \Lambda_{1}^{*} \\
& =\left(\Delta_{1}+\Delta_{1}^{*}\right)+\left(\Delta_{1} \circ j_{*}+\Lambda_{1}\right) \circ \Delta_{2}^{*} \circ \Lambda_{1}^{*}+\Lambda_{1} \circ \Delta_{2} \circ\left(j_{*} \circ \Delta_{1}^{*}+\Lambda_{1}^{*}\right) \\
& =\left(\Delta_{1}+\Delta_{1}^{*}\right)+\Delta_{2}^{*} \circ \Lambda_{1}^{*}+\Lambda_{1} \circ \Delta_{2} .
\end{aligned}
$$

Thus by (3.5), we see that $\Delta_{1} * \Delta_{2} \in \mathcal{V}$.

The associativity holds, since we have

$$
\begin{aligned}
\mathrm{id}-\left(\Delta_{1} * \Delta_{2}\right) \circ j_{*} & =\mathrm{id}-\left(\Delta_{1}+\left(\mathrm{id}-\Delta_{1} \circ j_{*}\right) \circ \Delta_{2}\right) \circ j_{*} \\
& =\left(\mathrm{id}-\Delta_{1} \circ j_{*}\right) \circ\left(\mathrm{id}-\Delta_{2} \circ j_{*}\right)
\end{aligned}
$$

and hence

$$
\begin{aligned}
\left(\Delta_{1} * \Delta_{2}\right) * \Delta_{3} & =\left(\Delta_{1}+\Lambda_{1} \circ \Delta_{2}\right)+\left(\Lambda_{1} \circ \Lambda_{2}\right) \circ \Delta_{3} \\
& =\Delta_{1}+\Lambda_{1} \circ\left(\Delta_{2}+\Lambda_{2} \circ \Delta_{3}\right) \\
& =\Delta_{1} *\left(\Delta_{2} * \Delta_{3}\right) .
\end{aligned}
$$

It is obvious that the zero homomorphism is variational and is the identity element.

Finally, for $\Delta \in \mathcal{V}$, its inverse $\Delta^{-1}$ is given by

$$
\Delta^{-1}=-\Lambda^{-1} \circ \Delta,
$$

where $\Lambda=\mathrm{id}-\Delta \circ j_{*}$ is an isometry of $\left(H_{2}(M), \cdot\right)$ by Lemma 3.4 This is seen as follows. We have

$$
\begin{aligned}
\left(-\Lambda^{-1} \circ \Delta\right) \circ j_{*} \circ\left(-\Lambda^{-1} \circ \Delta\right)^{*} & =\Lambda^{-1} \circ \Delta \circ j_{*} \circ \Delta^{*} \circ\left(\Lambda^{-1}\right)^{*} \\
& =\Lambda^{-1} \circ\left(\Delta+\Delta^{*}\right) \circ\left(\Lambda^{-1}\right)^{*} \\
& =\Lambda^{-1} \circ \Delta \circ\left(\Lambda^{-1}\right)^{*}+\Lambda^{-1} \circ \Delta^{*} \circ\left(\Lambda^{-1}\right)^{*} \\
& =-\Delta^{*} \circ\left(\Lambda^{-1}\right)^{*}-\Lambda^{-1} \circ \Delta \\
& =\left(-\Lambda^{-1} \circ \Delta\right)+\left(-\Lambda^{-1} \circ \Delta\right)^{*}
\end{aligned}
$$

by an argument similar to (3.3). Thus we have $\Delta^{-1}=-\Lambda^{-1} \circ \Delta \in \mathcal{V}$. Furthermore, since

we have

$$
\operatorname{id}-\left(-\Lambda^{-1} \circ \Delta\right) \circ j_{*}=\Lambda^{-1} \circ\left(\Lambda+\Delta \circ j_{*}\right)=\Lambda^{-1},
$$

and

$$
\Delta *\left(-\Lambda^{-1} \circ \Delta\right)=\Delta+\Lambda \circ\left(-\Lambda^{-1} \circ \Delta\right)=0
$$

$$
\left(-\Lambda^{-1} \circ \Delta\right) * \Delta=-\Lambda^{-1} \circ \Delta+\Lambda^{-1} \circ \Delta=0 .
$$

Thus $\mathcal{V}$ forms a group. This completes the proof.

Definition 3.6. A stable variational homomorphism of $M$ is a variational homomorphism $\Delta: H_{2}\left(M_{k}, \partial M_{k}\right) \rightarrow H_{2}\left(M_{k}\right)$ for some $k \geq 0$. Two stable variational homomorphisms $\Delta_{0}: H_{2}\left(M_{k_{0}}, \partial M_{k_{0}}\right) \rightarrow H_{2}\left(M_{k_{0}}\right)$ and $\Delta_{1}: H_{2}\left(M_{k_{1}}, \partial M_{k_{1}}\right) \rightarrow$ $H_{2}\left(M_{k_{1}}\right)$ of $M$ are said to be stably equivalent if

$$
\begin{aligned}
\Delta_{0} \oplus 0_{k_{0}^{\prime}}: H_{2}\left(M_{k_{0}+k_{0}^{\prime}}, \partial M_{k_{0}+k_{0}^{\prime}}\right) & \cong H_{2}\left(M_{k_{0}}, \partial M_{k_{0}}\right) \oplus H_{2}\left(\sharp_{0}^{\prime}\left(S^{2} \times S^{2}\right)\right) \\
\rightarrow H_{2}\left(M_{k_{0}+k_{0}^{\prime}}\right) & \cong H_{2}\left(M_{k_{0}}\right) \oplus H_{2}\left(\sharp^{k_{0}^{\prime}}\left(S^{2} \times S^{2}\right)\right)
\end{aligned}
$$


coincides with

$$
\begin{aligned}
& \Delta_{1} \oplus 0_{k_{1}^{\prime}}: H_{2}\left(M_{k_{1}+k_{1}^{\prime}}, \partial M_{k_{1}+k_{1}^{\prime}}\right) \cong H_{2}\left(M_{k_{1}}, \partial M_{k_{1}}\right) \oplus H_{2}\left(\sharp^{\prime}\left(S^{2} \times S^{2}\right)\right) \\
& \rightarrow H_{2}\left(M_{k_{1}+k_{1}^{\prime}}\right) \cong H_{2}\left(M_{k_{1}}\right) \oplus H_{2}\left(\sharp^{\prime}\left(S^{2} \times S^{2}\right)\right)
\end{aligned}
$$

for some $k_{0}^{\prime}$ and $k_{1}^{\prime}$ with $k_{0}+k_{0}^{\prime}=k_{1}+k_{1}^{\prime}$, where

$$
0_{k^{\prime}}: H_{2}\left(\sharp^{k^{\prime}}\left(S^{2} \times S^{2}\right)\right) \rightarrow H_{2}\left(\sharp^{k^{\prime}}\left(S^{2} \times S^{2}\right)\right)
$$

stands for the zero map. The set of all equivalence classes of stable variational homomorphisms $H_{2}\left(M_{k}, \partial M_{k}\right) \rightarrow H_{2}\left(M_{k}\right), k \geq 0$, of $M$ is called the stable variational group and is denoted by $\mathcal{S V}(M, \partial M)$. It is not difficult to see that $\mathcal{S} \mathcal{V}(M, \partial M)$ naturally forms a group, which is nothing but the inductive limit

$$
\lim _{k \rightarrow \infty} \mathcal{V}\left(M_{k}, \partial M_{k}\right)
$$

where we may naturally regard $\mathcal{V}\left(M_{k}, \partial M_{k}\right) \subset \mathcal{V}\left(M_{k+1}, \partial M_{k+1}\right)$ for each $k \geq 0$.

We have a natural map

$$
\Theta: \mathcal{S} \mathcal{M}(M, \partial M) \rightarrow \mathcal{S V}(M, \partial M)
$$

which maps each class of a stable diffeomorphism to the class of its variation map. This is a homomorphism of groups by Lemma 3.2(5).

Theorem 3.7. If $M$ is a smooth compact 1-connected 4-manifold with nonempty connected boundary, then the above correspondence $\Theta$ gives an isomorphism of groups.

Proof. The well-definedness and the injectivity follow from Theorem 2.2 ,

Let $\Delta: H_{2}\left(M_{k}, \partial M_{k}\right) \rightarrow H_{2}\left(M_{k}\right)$ be a stable variational homomorphism of $M$. Note that

$$
\Lambda=\mathrm{id}-\Delta \circ j_{*}: H_{2}\left(M_{k}\right) \rightarrow H_{2}\left(M_{k}\right)
$$

is an isometry of $\left(H_{2}\left(M_{k}\right), \cdot\right)$ by Lemma 3.4 .

The following lemma has been proved implicitly in the proof of Lemma 3.2 (see (3.4) ).

Lemma 3.8. $\Delta \beta \cdot \alpha=-\left(\Lambda^{-1} \circ \Delta \alpha\right) \cdot \beta$ for all $\alpha, \beta \in H_{2}\left(M_{k}, \partial M_{k}\right)$.

Let $V=M^{0} \cup_{\text {id }}\left(-M^{1}\right)$ with $M^{0}=M^{1}=M_{k}$ be the double of $M_{k}$, which is a smooth closed 1-connected 4-manifold. Furthermore, let $\delta: H_{2}\left(M_{k}, \partial M_{k}\right) \rightarrow$ $H_{2}(V)$ be the doubling homomorphism, which is given by

$$
\delta([a])=\left[i_{0} a-i_{1} a\right],
$$

where $a$ is an arbitrary 2-cycle of $\left(M_{k}, \partial M_{k}\right)$ and $i_{0}: M^{0} \rightarrow V$ and $i_{1}: M^{1} \rightarrow V$ are the inclusion maps. Then put

$$
J^{\prime}=\operatorname{im}\left(\delta+i_{1 *} \circ \Delta: H_{2}\left(M_{k}, \partial M_{k}\right) \rightarrow H_{2}(V)\right) .
$$

Furthermore, let $G(-\Lambda)$ denote the subgroup of $H_{2}\left(M^{0}\right) \oplus H_{2}\left(M^{1}\right)$ consisting of the elements of the form $(\alpha,-\Lambda \alpha), \alpha \in H_{2}\left(M^{0}\right)$, where we regard $\Lambda$ as an isomorphism from $H_{2}\left(M^{0}\right)$ to $H_{2}\left(M^{1}\right)$. Let $\iota: H_{2}\left(M^{0}\right) \oplus H_{2}\left(M^{1}\right) \rightarrow H_{2}(V)$ be the natural homomorphism induced by the inclusions. Then we have the following.

Lemma 3.9. $\iota G(-\Lambda) \subset J^{\prime}$. 
Proof. For every $\alpha \in H_{2}\left(M_{k}\right)$, we have

$$
\begin{aligned}
\left(\delta+i_{1 *} \circ \Delta\right)\left(j_{*} \alpha\right) & =\left(i_{0 *} \alpha-i_{1 *} \alpha\right)+i_{1 *} \circ \Delta \circ j_{*} \alpha \\
& =\left(i_{0 *} \alpha-i_{1 *} \alpha\right)+i_{1 *}(\alpha-\Lambda \alpha) \\
& =i_{0 *} \alpha-i_{1 *} \circ \Lambda \alpha .
\end{aligned}
$$

This shows our required inclusion.

Lemma 3.10. The subgroup $J^{\prime}$ of $H_{2}(V)$ is isotropic.

Proof. For every $\alpha, \beta \in H_{2}\left(M_{k}, \partial M_{k}\right)$, we have

$$
\begin{aligned}
& \left(\delta+i_{1 *} \circ \Delta\right)(\alpha) \cdot\left(\delta+i_{1 *} \circ \Delta\right)(\beta) \\
& =\delta \alpha \cdot \delta \beta+\delta \alpha \cdot\left(i_{1 *} \circ \Delta \beta\right)+\left(i_{1 *} \circ \Delta \alpha\right) \cdot \delta \beta+\left(i_{1 *} \circ \Delta \alpha\right) \cdot\left(i_{1 *} \circ \Delta \beta\right) \\
& =\Delta \beta \cdot \alpha+\Delta \alpha \cdot \beta-\Delta \alpha \cdot\left(j_{*} \circ \Delta \beta\right) \\
& =\left(\Delta \beta \cdot \alpha+\Delta^{*} \beta \cdot \alpha\right)-\left(\Delta^{*} \circ j_{*} \circ \Delta \beta\right) \cdot \alpha=0,
\end{aligned}
$$

since $\delta \alpha \cdot \delta \beta=0$ and $\Delta$ is variational. This completes the proof.

Consider the Mayer-Vietoris exact sequence for the pair $\left(M^{0}, M^{1}\right)$ :

$$
0 \longrightarrow H_{2}\left(\partial M_{k}\right) \longrightarrow H_{2}\left(M^{0}\right) \oplus H_{2}\left(M^{1}\right) \stackrel{\iota}{\longrightarrow} H_{2}(V) \stackrel{\partial^{\prime}}{\longrightarrow} H_{1}\left(\partial M_{k}\right) \longrightarrow 0 .
$$

Lemma 3.11. We have $\partial^{\prime} J^{\prime}=H_{1}\left(\partial M_{k}\right)$.

Proof. For every $\alpha \in H_{2}\left(M_{k}, \partial M_{k}\right)$, we have

$$
\partial^{\prime} \circ\left(\delta+i_{1 *} \circ \Delta\right) \alpha=\partial^{\prime} \circ \delta \alpha=\partial \alpha,
$$

where $\partial: H_{2}\left(M_{k}, \partial M_{k}\right) \rightarrow H_{1}\left(\partial M_{k}\right)$ is the boundary homomorphism in the exact sequence of the pair $\left(M_{k}, \partial M_{k}\right)$ :

$$
0 \longrightarrow H_{2}\left(\partial M_{k}\right) \longrightarrow H_{2}\left(M_{k}\right) \longrightarrow H_{2}\left(M_{k}, \partial M_{k}\right) \stackrel{\partial}{\longrightarrow} H_{1}\left(\partial M_{k}\right) \longrightarrow 0 .
$$

Since $\partial$ is surjective, we have the conclusion.

Let $J$ be a maximal isotropic subgroup of $H_{2}(V)$ containing $J^{\prime}$. Then by 3 , (4.2)] (see also [18]), there exists a smooth $h$-cobordism $W$ relative to boundary between $M^{0}$ and $M^{1}$ such that

$$
\operatorname{ker}\left(\kappa_{*}: H_{2}(V) \rightarrow H_{2}(W)\right)=J
$$

where $\kappa: V \rightarrow W$ is the inclusion map. Let $\eta: M^{0} \rightarrow M^{1}$ be the homotopy equivalence relative to boundary induced by the $h$-cobordism $W$. We can define the variation map $\Delta_{\eta}: H_{2}\left(M^{0}, \partial M^{0}\right) \rightarrow H_{2}\left(M^{1}\right)$ of $\eta$ as before.

Lemma 3.12. The variation map $\Delta_{\eta}$ coincides with $\Delta$.

Proof. Since $J^{\prime} \subset$ ker $\kappa_{*}$, we have

$$
-\kappa_{*} \circ \delta=\kappa_{*} \circ i_{1 *} \circ \Delta: H_{2}\left(M^{0}, \partial M^{0}\right) \rightarrow H_{2}(W) .
$$

Let $a$ be an arbitrary 2-cycle of $\left(M^{0}, \partial M^{0}\right)$. Then we have

$$
\begin{aligned}
\kappa_{*} \circ i_{1 *} \circ \Delta_{\eta}[a] & =\kappa_{*} \circ i_{1 *}([a-\eta a]) \\
& =\left[\kappa \circ i_{1} a-\kappa \circ i_{0} a\right] \\
& =\kappa_{*}(-\delta[a]) \\
& =\kappa_{*} \circ i_{1 *} \circ \Delta[a] .
\end{aligned}
$$


Since $\kappa_{*} \circ i_{1 *}: H_{2}\left(M^{1}\right) \rightarrow H_{2}(V)$ is an isomorphism, we have $\Delta_{h}=\Delta$. This completes the proof.

Now taking the connected sum along cobordisms of the smooth $h$-cobordism $W$ with the trivial cobordism $\left(\sharp^{k^{\prime}} S^{2} \times S^{2}\right) \times[0,1]$ for some $k^{\prime}$, we see that $\Delta$ is realized stably by a stable diffeomorphism of $M$ [11], 114. Hence the correspondence $\Theta$ is surjective. This completes the proof of Theorem 3.7.

Remark 3.13. Using an argument similar to the above together with the topological $h$-cobordism theorem of Freedman [5], we can also obtain some results about the topological mapping class group of topological 4-manifolds with boundary without stabilization. More precisely, let $M$ be a compact 1-connected topological 4-manifold with nonempty connected boundary. Let $\mathcal{M}^{\mathrm{TOP}}(M, \partial M)$ denote the group of topological isotopy classes relative to boundary of the homeomorphisms of $M$ which are the identity on the boundary. Then we have the natural map

$$
\Theta^{\mathrm{TOP}}: \mathcal{M}^{\mathrm{TOP}}(M, \partial M) \rightarrow \mathcal{V}(M, \partial M)
$$

which maps each isotopy class of a homeomorphism to its variation map. Then the argument in the proof of Theorem 3.7 shows that $\Theta^{\mathrm{TOP}}$ is surjective. We do not know if it is injective or not.

\section{Relation to STABle isometry GRoup}

In this section, we study the relationship between the stable variational group and the stable isometry group of a 4-manifold with boundary.

Definition 4.1. Let $M$ be a compact 4-manifold with boundary. We denote by $\mathcal{I}(M)$ the isometry group of the inner product space $\left(H_{2}(M), \cdot\right)$, where "." denotes the intersection form. A stable isometry of $M$ is an isometry of $\left(H_{2}\left(M_{k}\right), \cdot\right)$ for some $k$. Two stable isometries $\Lambda_{0}: H_{2}\left(M_{k_{0}}\right) \rightarrow H_{2}\left(M_{k_{0}}\right)$ and $\Lambda_{1}: H_{2}\left(M_{k_{1}}\right) \rightarrow H_{2}\left(M_{k_{1}}\right)$ of $M$ are said to be stably equivalent if the isometry $\Lambda_{0} \oplus$ id of $\left(H_{2}\left(M_{k_{0}+k_{0}^{\prime}}\right), \cdot\right)=$ $\left(H_{2}\left(M_{k_{0}}\right) \oplus H_{2}\left(\sharp k_{0}^{\prime} S^{2} \times S^{2}\right), \cdot\right)$ and the isometry $\Lambda_{1} \oplus$ id of $\left(H_{2}\left(M_{k_{1}+k_{1}^{\prime}}\right), \cdot\right)=$ $\left(H_{2}\left(M_{k_{1}}\right) \oplus H_{2}\left(\sharp^{k_{1}^{\prime}} S^{2} \times S^{2}\right), \cdot\right)$ coincide with each other for some $k_{0}^{\prime}$ and $k_{1}^{\prime}$ with $k_{0}+k_{0}^{\prime}=k_{1}+k_{1}^{\prime}$. The set of all equivalence classes of stable isometries of $M$ is called the stable isometry group of $M$ and is denoted by $\mathcal{S I}(M)$, which can naturally be identified with the inductive limit

$$
\lim _{k \rightarrow \infty} \mathcal{I}\left(M_{k}\right) .
$$

This obviously forms a group with respect to the composition of isometries.

Let $M$ be a 1-connected 4-manifold with nonempty connected boundary $\partial M=$ $K$. For each variational homomorphism $\Delta \in \mathcal{V}(M, \partial M)$, the endomorphism $\Lambda=$ id $-\Delta \circ j_{*}$ is an isometry of $\left(H_{2}(M), \cdot\right)$ by Lemma 3.4. Let us define

$$
\Xi: \mathcal{V}(M, \partial M) \rightarrow \mathcal{I}(M)
$$

by $\Xi \Delta=\Lambda$. By (3.6), $\Xi$ is a homomorphism of groups.

Let us denote by $\wedge^{2} H_{1}(K)^{*}$ the set of all skew-symmetric bilinear forms $H_{1}(K) \times$ $H_{1}(K) \rightarrow \mathbf{Z}$. Note that this naturally forms an additive group, which is finitely generated and free abelian. Note that an element $\kappa \in \wedge^{2} H_{1}(K)^{*}$ can also be 
regarded as a homomorphism $H_{1}(K) \rightarrow \operatorname{Hom}\left(H_{1}(K), \mathbf{Z}\right) \cong H^{1}(K) \cong H_{2}(K)$. For such an element $\kappa$, define $\widetilde{\kappa}: H_{2}(M, \partial M) \rightarrow H_{2}(M)$ by the composition

$$
H_{2}(M, \partial M) \stackrel{\partial}{\longrightarrow} H_{1}(K) \stackrel{\kappa}{\longrightarrow} H_{2}(K) \stackrel{\iota_{*}}{\longrightarrow} H_{2}(M),
$$

where $\partial: H_{2}(M, \partial M) \rightarrow H_{1}(K)$ is the boundary homomorphism and $\iota: K \rightarrow M$ is the inclusion. Then we can easily check that $\widetilde{\kappa}$ is a variational homomorphism and that the map $S: \wedge^{2} H_{1}(K)^{*} \rightarrow \mathcal{V}(M, \partial M)$ defined by $S \kappa=\widetilde{\kappa}$ is an injective homomorphism.

The main result of this section is the following.

Proposition 4.2. For a compact 1-connected 4-manifold $M$ with nonempty connected boundary $\partial M=K$, the sequence

$$
0 \longrightarrow \wedge^{2} H_{1}(K)^{*} \stackrel{S}{\longrightarrow} \mathcal{V}(M, \partial M) \stackrel{\Xi}{\longrightarrow} \mathcal{I}(M)
$$

is exact.

Proof. We have already observed that $S$ is injective.

It is easy to check that $\operatorname{im} S \subset \operatorname{ker} \Xi$. Suppose that $\Delta$ is an element of ker $\Xi$. Thus $\Xi \Delta=\mathrm{id}-\Delta \circ j_{*}$ is the identity of $H_{2}(M)$ and hence $\Delta=0$ on $\operatorname{im} j_{*}=\operatorname{ker} \partial$. Thus we may regard $\Delta$ as a homomorphism

$$
H_{2}(M, \partial M) / \operatorname{ker} \partial \cong H_{1}(K) \rightarrow H_{2}(M) .
$$

On the other hand, since $\Delta$ is variational, we have $\Delta+\Delta^{*}=\Delta \circ j_{*} \circ \Delta^{*}=0$. Hence, we have $0=\Delta \circ j_{*}+\Delta^{*} \circ j_{*}=\Delta^{*} \circ j_{*}$, which implies that $j_{*} \circ \Delta=0$. Thus im $\Delta \subset \operatorname{ker} j_{*}=H_{2}(K)$. Hence $\Delta$ can be regarded as a homomorphism $\kappa: H_{1}(K) \rightarrow H_{2}(K)$. Now it is easy to check that $\kappa \in \wedge^{2} H_{1}(K)^{*}$ and $\Delta=S \kappa$. This completes the proof.

We can characterize the image of $\Xi$ as follows. Let us first take a basis

$$
\left\{\alpha_{1}, \alpha_{2}, \ldots, \alpha_{u}, \alpha_{u+1}, \ldots, \alpha_{u+v}\right\}
$$

of $H_{2}(M)$ over the integers, where $u=\operatorname{rank} H_{2}(K), u+v=\operatorname{rank} H_{2}(M)$, and

$$
\left\{\alpha_{1}, \alpha_{2}, \ldots, \alpha_{u}\right\}
$$

is a basis of $\iota_{*} H_{2}(K)$. This is possible, since $\iota_{*} H_{2}(K)$ is a direct summand of $H_{2}(M)$. For $H_{2}(M, \partial M)$, we take the dual basis

$$
\left\{\alpha_{1}^{*}, \alpha_{2}^{*}, \ldots, \alpha_{u}^{*}, \alpha_{u+1}^{*}, \ldots, \alpha_{u+v}^{*}\right\}
$$

such that the intersection number in $M$ satisfies $\alpha_{k} \cdot \alpha_{l}^{*}=\delta_{k l}$, where

$$
\delta_{k l}= \begin{cases}1, & k=l \\ 0, & k \neq l\end{cases}
$$

Let

$$
\left(\begin{array}{ll}
A & B \\
C & D
\end{array}\right)
$$

be the matrix representation of a homomorphism $\Delta: H_{2}(M, \partial M) \rightarrow H_{2}(M)$ with respect to the above basis, where $A, B, C$ and $D$ are $u \times u, u \times v, v \times u$ and $v \times v$ integral matrices, respectively. Furthermore, we see easily that the matrix representation of $j_{*}$ with respect to the above basis must be of the form

$$
\widetilde{Q}=\left(\begin{array}{cc}
0 & 0 \\
0 & Q
\end{array}\right)
$$


for some nonsingular $v \times v$ integral matrix $Q$. Then it is easy to see that $\Delta$ is variational if and only if

$$
D+{ }^{t} D=D Q{ }^{t} D, A+{ }^{t} A=B Q^{t} B, B+{ }^{t} C=B Q{ }^{t} D .
$$

Thus, an isometry $\Lambda \in \mathcal{I}(M)$ is in the image of $\Xi$ if and only if, with respect to the above basis, $\Lambda$ is represented by a matrix of the form

$$
\left(\begin{array}{cc}
E_{u} & -B Q \\
0 & E_{v}-D Q
\end{array}\right)
$$

such that

$$
D+{ }^{t} D=D Q{ }^{t} D, A+{ }^{t} A=B Q{ }^{t} B, B+{ }^{t} C=B Q{ }^{t} D
$$

for some integral matrices $A, B, C$ and $D$, where $E_{w}$ denotes the $w \times w$ unit matrix.

If the diagonal entries of $Q$ are even, or if $H_{2}(K)=0$, then we can show that this condition is equivalent to that the following diagram is commutative:

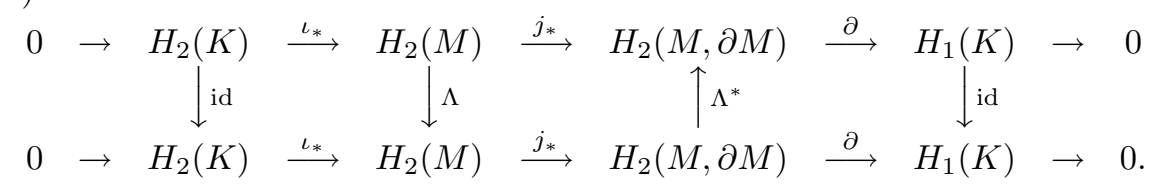

In this case, we have the exact sequence

$$
0 \longrightarrow \wedge^{2} H_{1}(K)^{*} \stackrel{S}{\longrightarrow} \mathcal{V}(M, \partial M) \stackrel{\Xi}{\longrightarrow} \mathcal{I}(M) \stackrel{\partial}{\longrightarrow} A_{\widetilde{Q}}(K) \longrightarrow 0,
$$

where $\partial$ and $A_{\widetilde{Q}}(K)$ are a homomorphism and a group as defined in [3, $\left.\S 1\right]$.

If some diagonal entries of $Q$ are not even and $H_{2}(K) \neq 0$, then the commutativity of the diagram (4.1) is still necessary, but it is not sufficient in general.

In fact, using Boyer's obstruction class $\theta[3$, we can show the following.

Proposition 4.3. An isometry $\Lambda \in \mathcal{I}(M)$ is in the image of $\Xi: \mathcal{V}(M, \partial M) \rightarrow$ $\mathcal{I}(M)$ if and only if $\partial \Lambda=\mathrm{id} \in A_{\widetilde{Q}}(K)$ and Boyer's obstruction $\theta(\mathrm{id}, \Lambda) \in I^{1}(K)$ vanishes, where $I^{1}(K)=\operatorname{im}\left(H^{1}(K) \rightarrow H^{1}\left(K ; \mathbf{Z}_{2}\right)\right)$.

Proof. Suppose $\partial \Lambda=$ id and $\theta(\mathrm{id}, \Lambda)=0$. Then by Boyer [3], there exists a homeomorphism $h: M \rightarrow M$ such that $\left.h\right|_{\partial M}=$ id and $h_{*}=\Lambda$. Let $\Delta=\Delta_{h} \in$ $\mathcal{V}(M, \partial M)$ be the variation map of $h$. Then it is easy to see that $\Xi \Delta=\Lambda$.

Conversely, if $\Lambda=\Xi \Delta$ for some $\Delta$, then we have already seen that $\partial \Lambda=\mathrm{id}$. Furthermore, by Remark 3.13, there exists a homeomorphism $h: M \rightarrow M$ such that $\left.h\right|_{\partial M}=\mathrm{id}$ and $\Delta_{h}=\Delta$. Hence by Boyer [3], the obstruction $\theta\left(\mathrm{id}, \Lambda_{h}\right)$ vanishes, where $\Lambda_{h}=h_{*}$. Since $\Lambda_{h}=\Xi \Delta_{h}=\Lambda$ by Lemma $3.2(2)$, we have $\theta(\mathrm{id}, \Lambda)=0$. This completes the proof.

Note that when the diagonal entries of $Q$ are even or $H_{2}(K)=0$, the obstruction $\theta(\mathrm{id}, \Lambda)$ always vanishes (see [3, (0.8) Proposition]).

The above proposition can also be proved purely algebraically by using the abovementioned characterization of the image of $\Xi$ in terms of matrices, together with the definition of the obstruction $\theta$.

All the above results hold stably as well. More precisely, we can naturally define the homomorphisms

$$
\mathcal{S} S: \wedge^{2} H_{1}(K)^{*} \rightarrow \mathcal{S} \mathcal{V}(M, \partial M), \mathcal{S} \Xi: \mathcal{S V}(M, \partial M) \rightarrow \mathcal{S I}(M)
$$


and the sequence

$$
0 \longrightarrow \wedge^{2} H_{1}(K)^{*} \stackrel{\mathcal{S} S}{\longrightarrow} \mathcal{S} \mathcal{V}(M, \partial M) \stackrel{\mathcal{S} \Xi}{\longrightarrow} \mathcal{S I}(M)
$$

is exact. The characterization of the image of $\Xi$ holds similarly for $\mathcal{S} \Xi$ as well.

\section{BOUNDARY-FREE DIFFEOMORPHISMS}

In this section, we study the stable mapping class group of boundary-free diffeomorphisms of compact 4-manifolds whose boundaries are certain spherical 3manifolds.

Definition 5.1. Let $M$ be a compact 4-manifold with boundary. Two stable diffeomorphisms $h_{0}: M_{k_{0}} \rightarrow M_{k_{0}}$ and $h_{1}: M_{k_{1}} \rightarrow M_{k_{1}}$ of $M$, which may not necessarily be the identity on the boundary, are said to be stably isotopic if the diffeomorphisms $h_{0} \sharp k_{0}^{\prime}(\mathrm{id}): M_{k_{0}+k_{0}^{\prime}} \rightarrow M_{k_{0}+k_{0}^{\prime}}$ and $h_{1} \sharp k_{1}^{\prime}(\mathrm{id}): M_{k_{1}+k_{1}^{\prime}} \rightarrow M_{k_{1}+k_{1}^{\prime}}$ are smoothly isotopic for some $k_{0}^{\prime}$ and $k_{1}^{\prime}$ with $k_{0}+k_{0}^{\prime}=k_{1}+k_{1}^{\prime}$. Note that the isotopy should leave the boundary invariant as a set, but can move points on the boundary. The set of all stable isotopy classes of stable diffeomorphisms of $M$ is called the stable mapping class group of boundary free diffeomorphisms of $M$ and is denoted by $\mathcal{S} \mathcal{M}(M)$. This obviously forms a group with respect to the composition of maps.

Let $\Lambda: H_{2}(M) \rightarrow H_{2}(M)$ be an isometry of a compact 4-manifold $M$ with boundary $K$. Then we have the commutative diagram

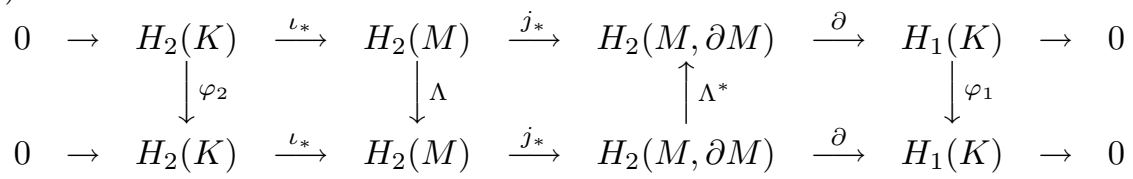

for some isomorphisms $\varphi_{1}$ and $\varphi_{2}$, where $\iota: \partial M \rightarrow M$ and $j: M \rightarrow(M, \partial M)$ denote the inclusions. Let $A(K)$ denote the group of automorphisms of $H_{*}(K)$, where an automorphism is required to preserve the torsion linking pairing $T_{1}(K) \times$ $T_{1}(K) \rightarrow \mathbf{Q} / \mathbf{Z}$ on the torsion subgroup $T_{1}(K)$ of $H_{1}(K)$ together with the intersection pairing $H_{1}(K) \times H_{2}(K) \rightarrow \mathbf{Z}$ (for details, see [3, §1]). Note that the isomorphism of $H_{*}(K)$ determined by $\left(\varphi_{1}, \varphi_{2}\right)$ in (5.1) is an automorphism and that the map

$$
\partial: \mathcal{I}(M) \rightarrow A(K)
$$

defined by $\partial \Lambda=\left(\varphi_{1}, \varphi_{2}\right)$ is a homomorphism.

Furthermore, let $H_{+}(K)$ denote the subgroup of $A(K)$ consisting of those automorphisms which are realized by an orientation preserving homeomorphism of $K$.

Set $\mathcal{I}^{0}(M)=\partial^{-1}\left(H_{+}(K)\right)$, which is a subgroup of $\mathcal{I}(M)$. It is easy to observe that we naturally have

$$
\mathcal{I}^{0}(M) \subset \mathcal{I}^{0}\left(M_{1}\right) \subset \cdots \subset \mathcal{I}^{0}\left(M_{k}\right) \subset \mathcal{I}^{0}\left(M_{k+1}\right) \subset \cdots .
$$

Thus we can define the inductive limit

$$
\mathcal{S I}^{0}(M)=\lim _{k \rightarrow \infty} \mathcal{I}^{0}\left(M_{k}\right)
$$

which is also a group. 
Note that if $H_{1}(K)=0$, then we have $\mathcal{S I}^{0}(M)=\mathcal{S I}(M)$. Note also that if $h: M_{k} \rightarrow M_{k}$ is an orientation preserving stable diffeomorphism of $M$, then $h_{*}: H_{2}\left(M_{k}\right) \rightarrow H_{2}\left(M_{k}\right)$ lies in $\mathcal{S I}^{0}(M)$.

The main result of this section is the following.

Theorem 5.2. Let $M$ be a smooth compact 1-connected 4-manifold with boundary $\partial M$ homeomorphic to a lens space $L(p, q)(p \geq 2)$, the 3 -sphere $S^{3}$, or the Poincaré homology 3 -sphere $\Sigma(2,3,5)$. Then the correspondence

$$
\Theta^{\text {fr }}: \mathcal{S M}(M) \rightarrow \mathcal{S I}^{0}(M)
$$

which maps each class of a stable diffeomorphism to the class of its induced isometry of the second homology group gives an isomorphism of groups.

Proof. Suppose that $h: M_{k} \rightarrow M_{k}$ is a stable diffeomorphism of $M$ such that $\Theta^{\mathrm{fr}} h$ is the identity. Then the automorphism $\left(\left.h\right|_{\partial M}\right)_{*}$ of $H_{*}(\partial M)$ must be the identity by a commutative diagram similar to (5.1). Then by results of [1, 2, 6], and [7] together with our assumption on the boundary, we see that $\left.h\right|_{\partial M}$ is smoothly isotopic to the identity. Thus, by extending the isotopy to the whole of $M$ using the collar neighborhood, we may assume that $\left.h\right|_{\partial M}$ is the identity. On the other hand, since $H_{1}(\partial M)$ is finite, the homomorphism $\Xi: \mathcal{V}\left(M_{k}, \partial M_{k}\right) \rightarrow \mathcal{I}\left(M_{k}\right)$ is injective by Proposition 4.2. Hence the variation map of $h$ must be the zero homomorphism. Thus by Theorem 2.2. $h$ is stably isotopic to the identity diffeomorphism (relative to boundary). Thus the homomorphism $\Theta^{\mathrm{fr}}$ is injective.

Let $\Lambda: H_{2}\left(M_{k}\right) \rightarrow H_{2}\left(M_{k}\right)$ be a stable isometry of $M$ such that $\Lambda \in \mathcal{I}^{0}\left(M_{k}\right)$. Then by the definition of $\mathcal{I}^{0}\left(M_{k}\right)$, there exists an orientation preserving homeomorphism $f: \partial M_{k} \rightarrow \partial M_{k}$ such that $\partial \Lambda=f_{*}$. We may assume that $f$ is a diffeomorphism (see [12]). Then by [3, (0.8) Proposition] together with the fact that $H_{*}(\partial M ; \mathbf{Q})=0$, we see that Boyer's obstruction $\theta(f, \Lambda)$ vanishes, which implies that there exists a homeomorphism $\widetilde{f}: M_{k} \rightarrow M_{k}$ such that $\widetilde{f}_{*}=\Lambda$ and $\left.\widetilde{f}\right|_{\partial M}=f$. Then by using the stable $h$-cobordism theorem [11, 14] together with an argument as in [3, §4] for constructing a smooth $h$-cobordism, we can show that there exists a diffeomorphism $\tilde{f}^{\prime}$ of $M_{k+k^{\prime}}$ for some $k^{\prime} \geq 0$ such that $\left.\tilde{f}^{\prime}\right|_{\partial M}=\left.\widetilde{f}\right|_{\partial M}$ and $\widetilde{f}_{*}^{\prime}$ and $\widetilde{f}_{*}$ are stably equivalent. Hence $\Theta^{\mathrm{fr}}$ is surjective. This completes the proof.

Remark 5.3. For the lens space $L(p, q), p \geq 2$, the structure of $H_{+}(L(p, q))$ has been determined. In fact, according to [2], $H_{+}(L(p, q))$ coincides with

$$
\begin{array}{ll}
\text { (1) }\{\mathrm{id}\}, & \text { if } p=2, \\
(2) \quad\{ \pm \mathrm{id}, \pm q\}, & \text { if } q^{2} \equiv 1 \quad(\bmod p) \text { and } q \not \equiv \pm 1 \quad(\bmod p), \\
(3) \quad\{ \pm \mathrm{id}\}, & \text { otherwise, }
\end{array}
$$

where $\pm q$ stands for the multiplication by $\pm q$. Thus, if $\partial M$ is diffeomorphic to $L(p, q)$ which satisfies $(1)$ or $(3)$, then $\mathcal{S I}^{0}(M)$ coincides with $\mathcal{S I}(M)$.

\section{REFERENCES}

[1] M. Boileau and J.-P. Otal, Groupe des difféotopies de certaines variétés de Seifert, C. R. Acad. Sc. Paris 303 (1986), 19-22. MR0849619 (87g:57022)

[2] F. Bonahon, Difféotopies des espaces lenticulaires, Topology 22 (1983), 305-314. MR0710104 (85d:57008)

[3] S. Boyer, Simply-connected 4-manifolds with a given boundary, Trans. Amer. Math. Soc. 298 (1986), 331-357. MR0857447 (88b:57023) 
[4] T. Cochran and N. Habegger, On the homotopy theory of simply connected four manifolds, Topology 29 (1990), 419-440. MR1071367 (91h:57006)

[5] M. Freedman, The topology of four-dimensional manifolds, J. Diff. Geom. 17 (1982), 357-453. MR0679066 (84b:57006)

[6] A. E. Hatcher, A proof of the Smale Conjecture, $\operatorname{Diff}\left(S^{3}\right) \simeq O(4)$, Ann. of Math. 117 (1983), 553-607. MR0701256 (85c:57008)

[7] C. Hodgson and J. H. Rubinstein, Involutions and isotopies of lens spaces, Knot theory and manifolds, Lect. Notes Math. no. 1144, 1985, Springer, Berlin, Heidelberg, New York, Tokyo, pp. 60-96. MR0823282 (87h:57028)

[8] L. Kauffman, Branched coverings, open books and knot periodicity, Topology 13 (1974), 143-160. MR0375337 (51:11532)

[9] M. Kreck, Isotopy classes of diffeomorphisms of $(k-1)$-connected almost-parallelizable $2 k$ manifolds, Algebraic topology, Aarhus 1978 (Proc. Sympos., Univ. Aarhus, Aarhus, 1978), pp. 643-663, Lecture Notes in Math., 763, Springer-Verlag, Berlin, 1979. MR0561244 (81i:57029)

[10] M. Kreck, $h$-cobordisms between 1-connected 4-manifolds, Geometry and Topology 5 (2001), 1-6. MR.1812433 (2002d:57028)

[11] T. Lawson, Trivializing 5-dimensional h-cobordisms by stabilization, Manuscripta Math. 29 (1979), 305-321. MR0545047 (80i:57024)

[12] E. E. Moise, Geometric topology in dimensions 2 and 3, Graduate Texts in Mathematics, Vol. 47, Springer-Verlag, New York, Heidelberg, 1977. MR0488059 (58:7631)

[13] B. Perron, Pseudo-isotopies et isotopies en dimension quatre dans la catégorie topologique, Topology 25 (1986), 381-397. MR0862426 (89g:57024)

[14] F. Quinn, The stable topology of 4-manifolds, Topology Appl. 15 (1983), 71-77. MR0676968 (84b:57023)

[15] F. Quinn, Isotopy of 4-manifolds, J. Diff. Geom. 24 (1986), 343-372. MR0868975 (88f:57020)

[16] O. Saeki, Theory of fibered 3 -knots in $S^{5}$ and its applications, J. Math. Sci. Univ. Tokyo 6 (1999), 691-756. MR1742599 (2001b:57058)

[17] O. Saeki, Open books on 5-dimensional manifolds, Hiroshima Math. J. 32 (2002), 189-205. MR:1925897 (2003j:57042)

[18] C. T. C. Wall, On simply-connected 4-manifolds, J. London Math. Soc. 39 (1964), 141-149. MR0163324 (29:627)

Faculty of Mathematics, Kyushu University, Hakozaki, Fukuoka 812-8581, Japan

E-mail address: saeki@math.kyushu-u.ac.jp 